\title{
Improvement and Verification for Combined Positioning Algorithm Based on UWB and Inertial Navigation
}

\author{
Hongliang Zhao ${ }^{1, a}$, Shaofu Gao ${ }^{1, b}$ and Weiyan $\mathrm{Hou}^{1, \mathrm{c}^{*}}$ \\ ${ }^{1}$ School of Information Engineering, Zhengzhou University, Zhengzhou 450001, China \\ azhaohongliang360@163.com, bgsf123@foxmail.com, ciewyhou@zzu.edu.cn \\ *The corresponding author
}

Keywords: Ultra wide band (UWB); Inertial navigation system; Trusted factors; Fitting thought

\begin{abstract}
In order to meet requirements of three-dimensional (3D) indoor positioning in the environment with multipath interference and non-line-of-sight (NLOS) errors, this paper improves setting rules of trusted factors about ultra wide band (UWB) and the inertial navigation system (INS) to the indoor positioning algorithm, realizing confidence factors' dynamic calibration. A method to restrain NLOS errors is proposed, and the fitting method is used to smooth singular points in the track. An actual system is built to verify the improved performance of this algorithm. The actual tests show that the algorithm can effectively improve the 3 dimensional positioning accuracy, positioning error can be controlled within $20 \mathrm{~cm}$.
\end{abstract}

\section{Introduction}

UWB technology has become a hot research topic in recent years due to its advantages such as high speed, low power consumption and high penetration. While in scenes such as serious multipath interference and the non line of sight error (Non-Line-of-Sight, NLOS), UWB positioning effects are not obvious, and the accuracy of tests can only reaches meter levels [1].

The prominent advantage of the inertial navigation system is the independence and continuity in the use of navigation and positioning. Using the nine axis inertial navigation module of the collocation magnetometer and accelerometer, motion information of the object can be measured accurately, and the direction and distance of the motion of the object can be calculated by the track [2]. But it also has some problems, for example, drift errors of gyroscope and magnetometer are vulnerable to electromagnetic interference to obtain accurate heading. In addition, the dead reckoning error integral inherent mechanism of cumulative effect, so it limits the time of the application of inertial navigation [3].

Previous scholars studied the application of positioning algorithm of weighted fusion for ranging and positioning through combining Zigbee and inertial navigation. By setting Zigbee's and navigation module's confidence factors, this method expected to obtain more precise location data. While the factor's trusting degree is to set to a fixed value, so it can not do timely feed back and adjust of the weighted algorithm. And in doors without shelters, the average location accuracy is just 0.8 meter [4]. In order to meet the positioning requirement of higher accuracy and more complex indoor environments, it also needs to continue to explore stronger anti-interference methods and improved algorithms.

\section{Optimization Methods}

Whole Thought of Optimization Methods in this Paper. A combined positioning algorithm is applied in this paper to adjust positioning accuracy in this paper. By the known initial position of the starting position data of the anchor nodes, target testing nodes are initialized in the process of moving to obtain the UWB valuation by Time of Arrival (TOA) method [5], and using of the nine axis navigation modules of the testing nodes' starting position data to measure lengths and aerial angles. Then calculate by the Kalman filter and make timely adjustments to the displacement and velocity errors to the normal range [6]. It is assumed that testing nodes move in a straight line in 
short time, position data of testing nodes are calculated in the next moment by using the plane geometry method. Finally, using the fitting algorithm for singular points smoothing, we can get final joint positioning data of moving nodes [7].

The general formula of the combined algorithm is as follows

$$
\operatorname{loc}_{\text {end }}=\frac{\alpha}{\alpha+\beta} \operatorname{loc}_{U W B}+\frac{\beta}{\alpha+\beta} \operatorname{loc}_{\text {Navi }}
$$

Among Eq. 1, loc end expresses as final position data of a certain moment of a testing node, loc $_{U W B}$ defines as positioning data for the location data by the UWB module to calculate, $\operatorname{loc}_{\text {Navi }}$ is the inertial navigation calculating data, $\alpha$ and $\beta$ are defined as trust factors about UWB and the inertial navigation in the joint positioning system $(0 \leq \alpha \leq 1,0 \leq \beta \leq 1)$.

UWB Trusted Factor- $\alpha$ 's Numeric Setting Rule. Eq. 1 is of the $\alpha$ 's setting rule for UWB joint positioning reliability factor. When the average value is larger than the line spacing of anchor nodes far $1 / 2$ the distance from the anchor node to the target node, $\alpha$ 's value is invalid. At the moment, $\alpha$ will be calculated from the start of 0.5 . The value of $\alpha$ is

$$
\alpha=\left\{\begin{array}{c}
\frac{d_{\text {ave }}}{D_{\max }}, d_{\text {ave }} \leq D_{\text {max }} \\
0, d_{\text {ave }}>D_{\max }
\end{array}\right.
$$

Among Eq. 2, $\mathrm{d}_{\mathrm{ave}}$ is the average value of the straight line distance between anchor nodes and the testing node, and $\mathrm{D}_{\max }$ is half of the distance between anchor nodes.

The Reduction and Optimization Measures of the Inertial Navigation Module's Cumulative Error. In order to reduce the cumulative error of the inertial navigation module, it is proposed that once the testing node is at zero speed, and the angular velocity of the gyroscope, the acceleration of the accelerometer and its variance are all within the threshold value, the target node is considered as in a static state. Once the testing node is detected at zero speed, the positioning system will carry on Kalman filtering, $\beta$ in Eq. 1 will be restored to the initial value, then the error of displacement and velocity will be adjusted to the normal range [8]. Fig. 1 is the specific flow chart for the detection of calculating $\beta$ 's value and the state of inertial navigation module.

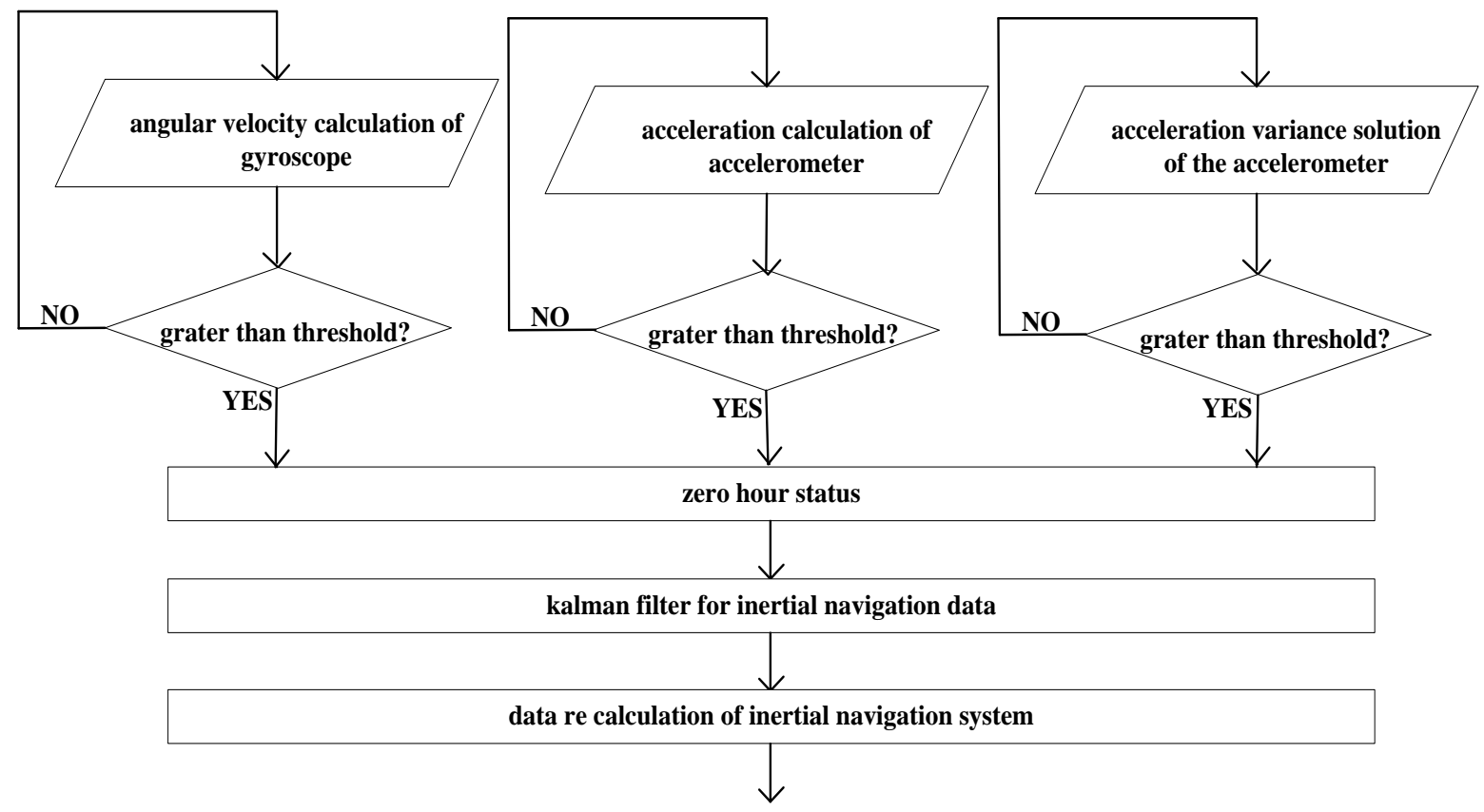

Figure 1. Flow chart of optimizing the inertial navigation algorithm

Smoothing of Singular Points. After calculating the testing node's positioning data by the algorithm described above, there are also some singular points. In this paper, we use the linear least 
square's method to estimate the overall changing trend of testing nodes' direction of motion to reduce the influence of singular points on the positioning accuracy [9].

In the fitting calculation, the linear least square criterion is used to determine a set of undetermined coefficients $a_{1}, a_{2}, \ldots a_{m}$, making the square of the distance about $\delta_{i}$ between the point $\left(x_{i}, y_{i}\right)(1 \leq i \leq \mathrm{n})$ and the curve $\mathrm{y}=f(x)$ to reach the minimum [10]. The calculation formula is as follows

$$
J\left(a_{1}, a_{2}, \cdots, a_{m}\right)=\sum_{i=1}^{n} \delta_{i}^{2}=\sum_{i=1}^{n}\left[f\left(x_{i}\right)-y_{i}\right]^{2}=\sum_{i=1}^{n}\left[\sum_{i=1}^{m} a_{k} r_{k}\left(x_{i}\right)-y_{i}\right]^{2}
$$

Moving track effects of the testing node before and after fitting is shown in Fig. 2.

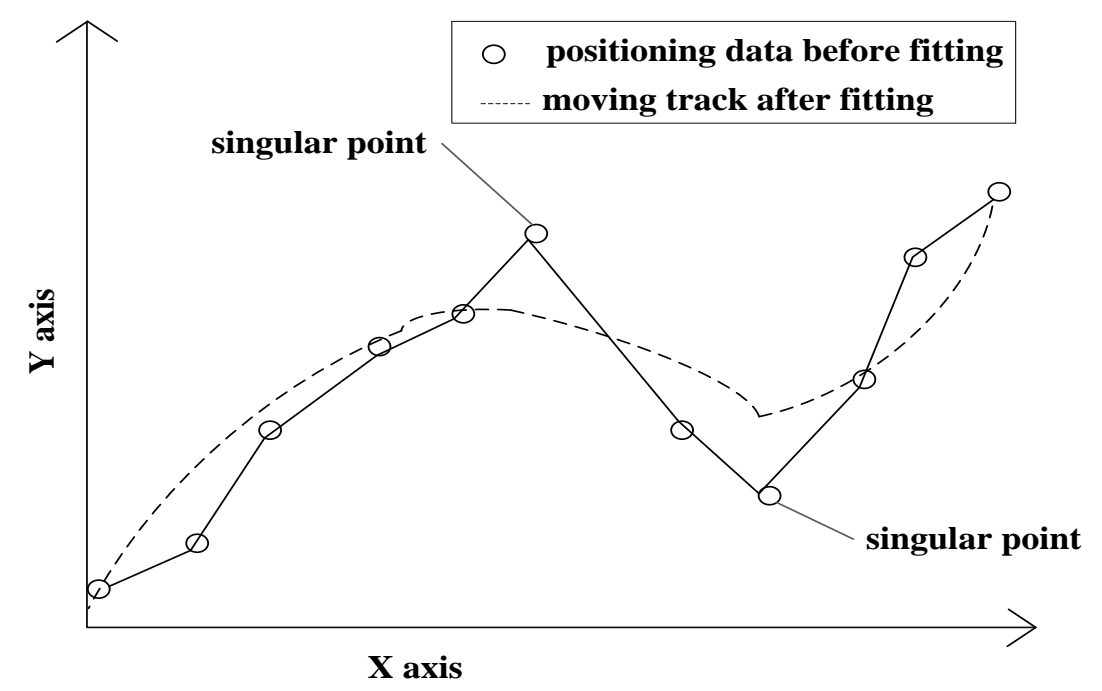

Figure 2. Mobile trajectory before and after fitting process

\section{Software and Hardware Testing Platform}

In order to test the effect of the improved measurement in this paper, the testing platform is designed and implemented as shown in Fig. 3. This platform includes four anchor nodes, some testing nodes, a wireless router and an upper computer. Anchor nodes should be firstly fixed around the test environment and setting one of anchor nodes as the origin. In the tracking process, target nodes only transfer real-time measurement data calculating by UWB and inertial navigation to the upper computer through the wireless router, then the upper computer calculates positioning data and does the fitting process [11]. Finally, real-time positioning data of testing nodes are displayed on the upper computer.

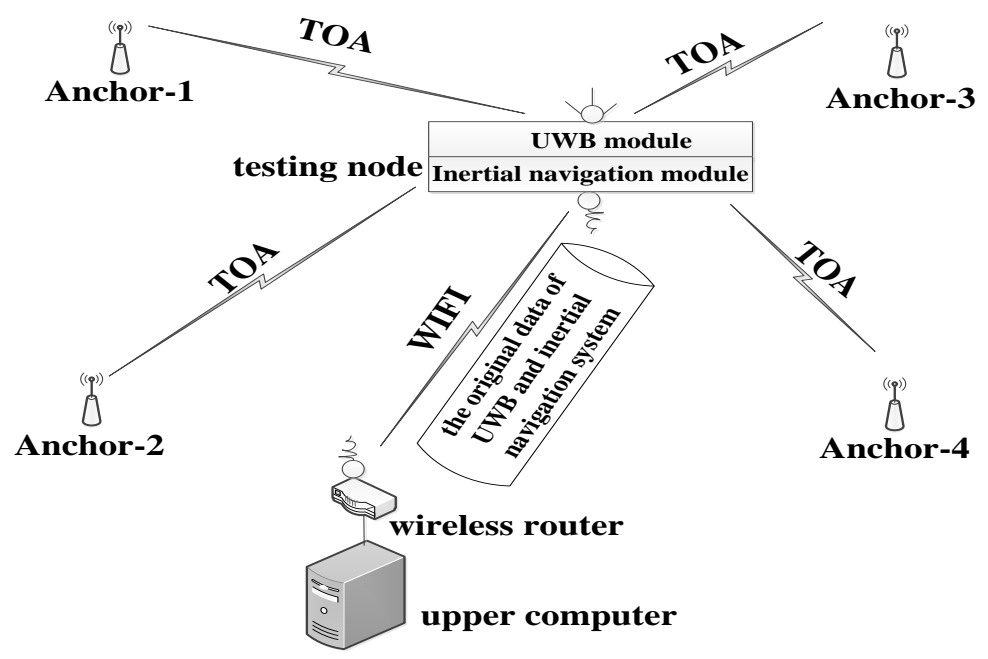

Figure 3. Diagram of the testing platform 


\section{Testing Results and Analyses}

As comparing with positioning effects of using the method in this paper and only using the UWB module, a testing node moved slowly in the testing room to record the $3 \mathrm{D}$ distance to the origin, and comparing with the actual distance. It can be seen from Table 1 that when only using UWB module to calculate the positioning data, the positioning accuracy was less than ideal due to the influence of multipath interference and NLOS error, even the maximum error reached 2 meters. While after using the method in this paper, the 3D positioning error reduced within 20 centimeters.

Table 1 Comparison of the effect of combined positioning and only UWB positioning

\begin{tabular}{|c|c|c|}
\hline $\begin{array}{c}\text { The actual coordinates of the } \\
\text { target node from the origin } \\
\text { (unit: }[\mathrm{m}])\end{array}$ & $\begin{array}{c}\text { UWB positioning of the target } \\
\text { node distance from the origin of } \\
\text { the test coordinates (unit: [m] })\end{array}$ & $\begin{array}{c}\text { Joint positioning of the target node } \\
\text { distance from the origin of the test } \\
\text { coordinates (unit: [m] })\end{array}$ \\
\hline$(0.00,3.50,1.00)$ & $(0.27,3.32,0.89)$ & $(0.19,3.46,1.08)$ \\
\hline$(0.00,5.50,1.00)$ & $(0.21,5.29,0.86)$ & $(0.10,5.59,0.92)$ \\
\hline$(1.00,5.00,1.50)$ & $(0.63,5.12,1.79)$ & $(0.89,5.16,1.39)$ \\
\hline$(1.00,7.00,1.50)$ & $(0.77,6.53,1.21)$ & $(1.16,7.13,1.56)$ \\
\hline$(3.00,8.00,1.70)$ & $(3.59,7.32,1.43)$ & $(3.13,8.92,1.65)$ \\
\hline$(3.00,13.00,1.50)$ & $(3.22,11.19,0.96)$ & $(3.20,12.81,1.32)$ \\
\hline$(6.00,15.00,1.00)$ & $(6.89,14.27,0.72)$ & $(5.83,15.19,1.16)$ \\
\hline$(6.00,17.00,1.50)$ & $(7.26,15.39,1.32)$ & $(6.11,16.81,1.57)$ \\
\hline
\end{tabular}

For comparing with tracking recording effects of with and without fitting process, a testing node moved at the speed of $0.5 \mathrm{~m} / \mathrm{s}$ in the testing room, MATLAB recorded its trajectory data once per 0.3 second, the track record without fitting process was shown as in Fig. 4, the track record with fitting process was shown as in Fig. 5. From Fig. 4, we can see that there were many singular points in the trajectory, and it should take a long time to correct the error of singular points to the normal range. While from Fig. 5, it can be seen that although there were still some singular points, the error could be quickly fitted to the calibration, adjusting time is smaller, and the trajectory is more smooth.

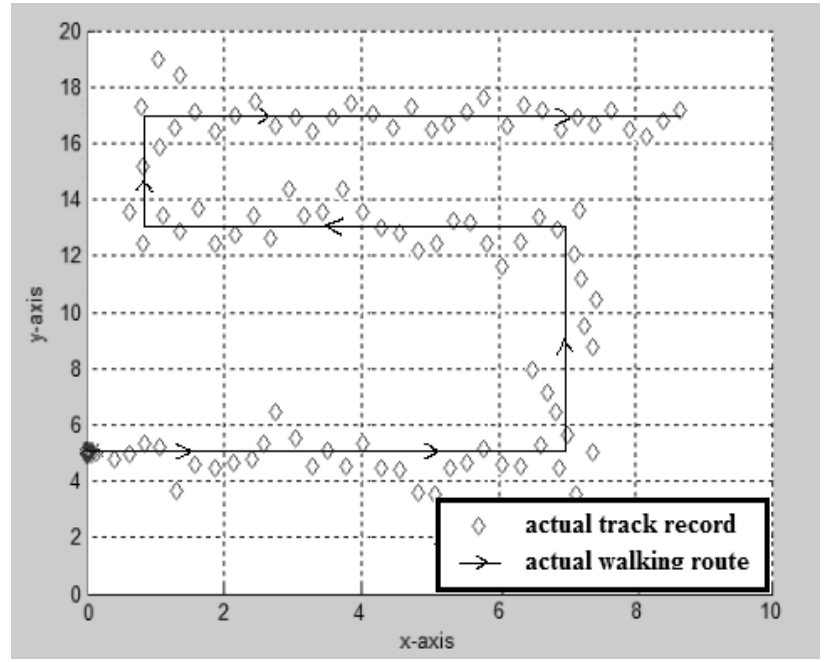

Figure 4. Track record without fitting process

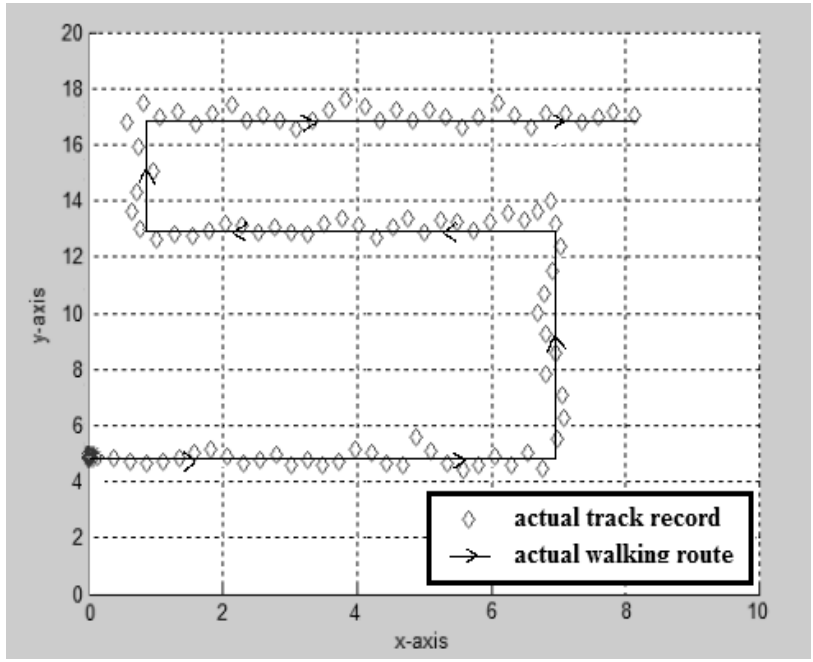

Figure 5. Track record with fitting process

\section{Summary}

An algorithm of combining of UWB and strapdown inertial navigation system for high precision 3D localization in complex environments is proposed in this paper. The algorithm combines with the 
idea of the track estimation and the fitting thought of the location point based on linear least squares method, the dependable factors of UWB and inertial navigation system for single location are defined, and these dependable factors are automatically weighted by using the thought of weighted average to determine the final location. Through tests, it is improved that this method effectively improves the 3D positioning accuracy in the more serious multipath interference and NLOS error in indoor scene target node. In order to achieve more accurate positioning, wider applications, subsequent research intends to GPS active positioning system integrated into the platform.

\section{Acknowledgements}

This paper's authors would like to express appreciation of financial support by National Natural Science Foundation of China (61573237) and Henan Provincial Science and Technology Department in 2015 Key Projects of Science and Technology (152102210344).

\section{References}

[1] J. Fu: Ship Electronic Engineering, Vol. 29 (2009) No. 1, p. 76 (In Chinese).

[2] Z.M. Li, F.Y. Duan and Z.P. Li: Transducer and Microsystem Technologies, Vol. 31 (2012) No. 6, p. 94 (In Chinese).

[3] Z.Y. Gao: Inertial Navigation System Technology (Science Press, China, 2012).

[4] L. Zhou, Y.T. Fu and G.J. Li: Application of Electronic Technique, Vol. 40 (2014) No. 4, p. 73 (In Chinese).

[5] H. Yu, G. Huang and J. Gao: IEEE Transactions on Wireless Communications, Vol. 11 (2012) No. 1, p. 44.

[6] M. Yang: Research on Small Strap Inertial Navigation System (MS., Central South University, China 2010), p. 21 (In Chinese).

[7] Y, He, Y. Liu and X. Shen, IEEE Transactions on Mobile Computing, Vol. 12 (2013) No. 2, p. 333.

[8] H. Yin, H. Guo: Science of Surveying and Mapping, Vol. 39 (2014) No. 1, p. 21 (In Chinese).

[9] Z.Q. Xia, A. Dai and P. Li: Computer Engineering and Applications, Vol. 51 (2015) No. 17, p. 102 (In Chinese).

[10] Z.Q. Jiao, W.L. Xiong, L. Zhang: Journal of Southeast University, Vol. 38 (2008) No. A1, p. 249 (In Chinese).

[11] X.Q. Chen, M. Wang and F. Gao: Microcomputer Information, Vol. 25 (2009) No. 25, p 132 (In Chinese). 